\title{
Mass Barcode Signal Amplification for Multiplex Allergy Diagnosis by MALDI-MS
}

\author{
Xiaoqin Zhong, ${ }^{\dagger}$ Liang Qiao, ${ }^{\dagger}{ }^{\dagger}$ Natalia Gasilova, ${ }^{\dagger}$ Baohong Liu, ${ }^{\ddagger}$ and Hubert H. Girault* ${ }^{\dagger}$ \\ ${ }^{\dagger}$ Laboratoire d’Electrochimie Physique et Analytique, Ecole Polytechnique Fédérale de Lausanne, Rue de l'industrie 17, CH-1951 \\ Sion, Switzerland \\ ${ }^{\ddagger}$ Chemistry Department, Fudan University, 220 Handan Road, 200433 Shanghai, China
}

Supporting Information

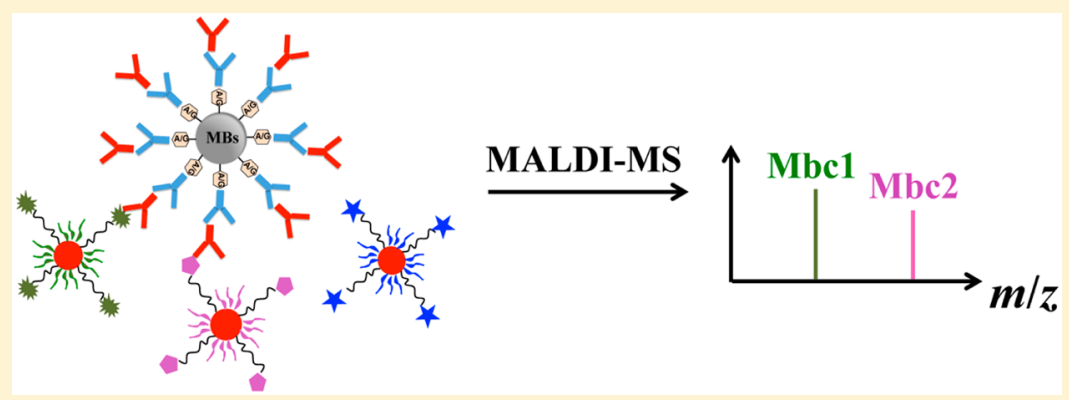

ABSTRACT: A highly sensitive method based on mass-barcoded gold nanoparticles (AuNPs) and immunomagnetic separation has been developed for multiplex allergy diagnosis by MALDI mass spectrometry in a component-resolved manner. Different analytical probes were prepared by coating AuNPs with individual allergenic proteins and mass barcode, represented by polyethylene glycol molecules of various chain lengths. Magnetic beads (MBs) functionalized with antihuman IgE antibodies (Abs) were used as immunomagnetic capture probes. IgE Abs were extracted from a patient's blood serum by the formation of a sandwich structure between the AuNPs and MBs. Multiple specific IgE Abs were simultaneously identified by mass spectrometry detection of the mass barcodes, providing an efficient component-resolved allergy diagnosis. Because of the signal amplification provided by the mass barcodes, the developed diagnosis method is very sensitive, with a limit of detection down to picograms per milliliter level for specific IgE Abs. The method can be potentially useful when the sample amount is highly limited and a multiplex diagnostic procedure is required.

$\mathrm{M}$ ass barcode-based mass spectrometry (MS) signal amplification is a technique widely utilized in DNA assays, ${ }^{1,2}$ immunoassays, ${ }^{3,4}$ and tissue imaging. ${ }^{5,6}$ Mass barcodes, which are small tag molecules and easily detectable by MS, are grafted on nano/micro-particles in large excess together with the large target biomolecules. The detection of these tag molecules results in an amplified signal of the targets. For immunoassays, ${ }^{3,4,7}$ the mass barcodes have been utilized to probe the IgG antibodies (Abs)-involved immune binding events on a biochip or a gold plate, which acted as a flat surface to capture and separate the antigens from bulk solutions. This surface-based separation approach requires a multiple-step procedure, which is rather cumbersome and time-consuming. Besides, the full accessibility of antigens to the Abs, which are preimmobilized on a $2 \mathrm{D}$ surface, is also limited.

Meanwhile, commercially available micromagnetic beads (MBs) with immunoaffinity coating are more advantageous for fast and convenient isolation of the target molecules. They possess large active surface area and are easy in manipulation. The immunoreactions can be performed in a homogeneous way through gentle mixing of MBs with other reagents and may result in a faster target-binding process. Optimization of the target enrichment for sensitive analysis can also be easily realized by adjusting the MBs amount and the final sample volume applied for analysis. Therefore, the combination of MBs-based immunoassays with mass barcode signal amplification protocol can be promising for MS detection of large biomolecules such as allergens.

Allergies, especially the ones caused by food products and plant pollen, are a constantly growing health problem worldwide. ${ }^{8}$ As pollen and foodstuff often contain multiple allergenic proteins that can provoke allergic reactions for a patient, component resolved diagnosis (CRD) should be performed. ${ }^{8}$ Allergy CRD is typically performed with individual purified allergenic proteins to detect the level of specific $\operatorname{IgE}$ Abs in human blood serum by enzyme-linked immunosorbent assay (ELISA). ${ }^{9}$ Commercially available ELISA-based kits ${ }^{10}$ or automated platforms for allergy CRD usually require 20-50 $\mu \mathrm{L}$ of blood serum and provide the limit of detection (LOD) for specific IgE Abs as low as $0.24 \mathrm{ng} / \mathrm{mL}(0.1 \mathrm{IU} / \mathrm{mL})$ level $^{11}$ Fluorophore-labeled immunoassay, ${ }^{12}$ magnetophoretic immu-

Received: March 22, 2016

Accepted: May 31, 2016

Published: May 31, 2016 


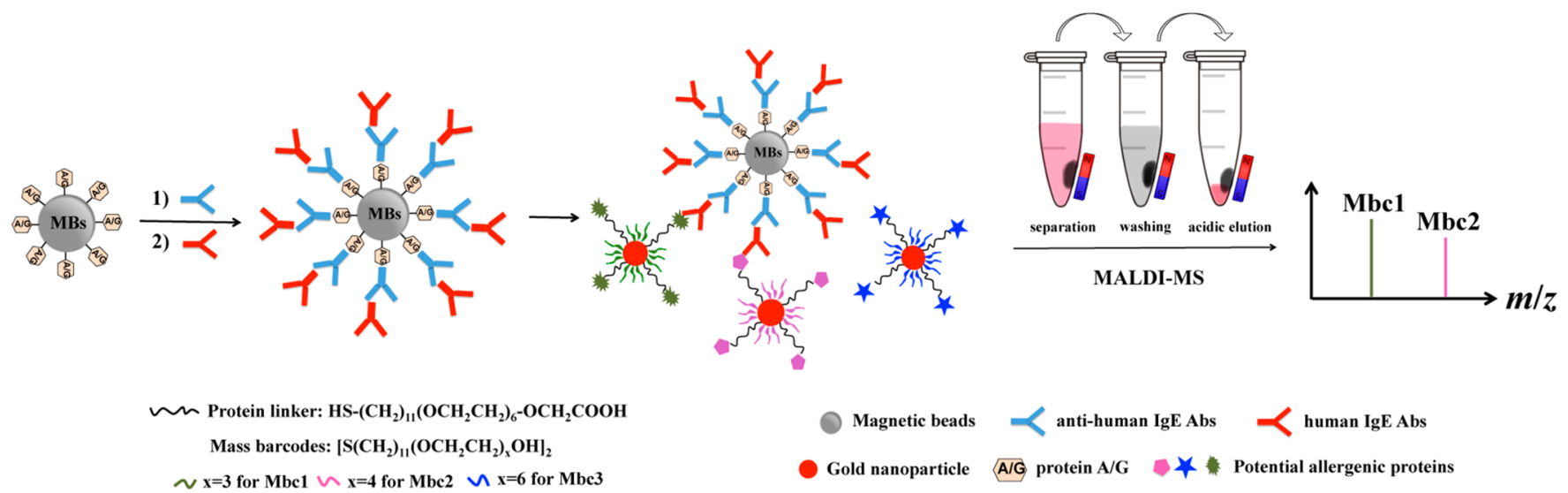

Figure 1. Schematic representation of coupling mass-barcoded AuNPs probes with immunomagnetic separation for simultaneous diagnosis of multiple allergic reactions via detection of specific IgE Abs. MALDI-MS: matrix-assisted laser desorption/ionization mass spectrometry; MBs: magnetic beads; Mbc: mass barcode.

noassay, ${ }^{13}$ interferometric reflectance imaging sensors, ${ }^{14}$ and immunoaffinity capillary electrophoresis (IACE) coupled with MS detection, ${ }^{15}$ were also developed. However, in the listed analytical methods, the allergy diagnosis usually relies on expensive enzyme or fluorescent labels. Special devices, such as the antigen array immobilized on a $2 \mathrm{D}$ surface, the microfluidic chip, or the separation capillary preloaded with affinity probes, are also required for these techniques, making them quite complex and laborious. Regarding these factors above, the simplification of the device, improvement of sensitivity and reduction of sample consumption are still needed and under development for the analysis of specific IgE Abs in the allergic patient's blood serum.

Here, we employ a mass barcode amplification strategy combined with commercial protein $\mathrm{A} / \mathrm{G}$ coated-MBs (protein $\mathrm{A} / \mathrm{G} @ \mathrm{MBs}$ ) to enhance the simplicity and sensitivity of the multiplex allergy CRD with MS detection as displayed in Figure 1. Polyethylene glycol molecules with different chain lengths were chosen as mass barcodes and grafted onto gold nanoparticles (AuNPs) that were further modified with specific allergenic proteins to form AuNPs probes. At the same time, protein A/G@MBs were modified with antihuman IgE Abs and formed a sandwich structure with specific $\operatorname{IgE} \mathrm{Abs}$ from patient's blood serum and AuNPs probes. The MS detection of the mass barcodes from AuNPs indicated the presence of respective specific IgE Abs and allowed allergy CRD in a highly sensitive manner. LOD of $18 \mathrm{pg} / \mathrm{mL}(7.5 \mathrm{mIU} / \mathrm{mL})$ for specific $\operatorname{IgE} \mathrm{Abs}$ was achieved for the analysis of antibeta-lactoglobulin Abs in model experiments, allowing using afterward only $1 \mu \mathrm{L}$ of blood serum from allergic patient for $\mathrm{CRD}$ diagnosis.

\section{EXPERIMENTAL SECTION}

Allergy CRD with the Patient's Blood Serum. First, the citrate-stabilized AuNPs were derivatized with the mixed thiolcontaining oligomers of mass barcodes and protein linkers. Various allergenic proteins-conjugated AuNPs probes were then produced by immobilizing individual purified cow's milk proteins, including lactoferrin, bovine serum albumin (BSA), $\beta$ lactoglobulin B ( $\beta$-lac B), $\alpha$-casein, $\beta$-casein, and $\kappa$-casein, onto the AuNPs with distinct mass barcodes covalently. More details about the used reagents, synthesis procedure of AuNPs and mass-barcoded AuNPs probes, and their relevant characterizations are given in Supporting Information, SI-1, SI-2, and SI3.
The antihuman IgE Abs-modified MBs were prepared by incubating $10 \mu \mathrm{L}$ of $10 \mathrm{mg} / \mathrm{mL}$ protein A/G@MBs with $5 \mu \mathrm{L}$ of $1 \mathrm{mg} / \mathrm{mL}$ antihuman $\mathrm{IgE}$ Abs for $30 \mathrm{~min}$ at room temperature (RT). Blood serum of a milk allergic patient was always diluted with $10 \mathrm{mM}$ phosphate buffer ( $\mathrm{PB}, \mathrm{pH}$ 7.4) prior to the diagnosis. Initial required volume of the serum varied from 50 to $1 \mu \mathrm{L}$, depending on the dilution factor, changing from 10 up to 100000 times. The modified MBs were then added to $300 \mu \mathrm{L}$ of the diluted blood serum to extract human IgE Abs through $30 \mathrm{~min}$ of incubation at RT. The supernatant was discarded and the protein A/G-antihuman IgE Abs-human IgE Abs immunocomplex formed on $\mathrm{MBs}$ was resuspended into $10 \mu \mathrm{L}$ of $10 \mathrm{mM} \mathrm{PB}, \mathrm{pH} 7.4$, for subsequent use.

Allergy diagnosis was first performed with each type of the allergen-conjugated AuNPs separately. A total of $20 \mu \mathrm{L}$ of appropriate protein-conjugated AuNPs (15 nM AuNPs) were mixed with $10 \mu \mathrm{L}$ of the prepared human IgE-based immunocomplex on MBs. The mixture was diluted to $300 \mu \mathrm{L}$ with $10 \mathrm{mM}$ PB and incubated for $30 \mathrm{~min}$ at RT. After magnetic separation step, acidic elution and MALDI-MS detection of captured allergen-coated AuNPs were carried out as described in SI-4. Multiplex allergy diagnosis was realized by adding simultaneously various AuNPs probes $(20 \mu \mathrm{L}$ and 15 $\mathrm{nM}$, each) into $10 \mu \mathrm{L}$ of the human IgE-captured MBs. The mixture was diluted to $300 \mu \mathrm{L}$ with $10 \mathrm{mM} \mathrm{PB}$ and incubated for $30 \mathrm{~min}$ at RT, followed by magnetic separation step, AuNPs elution, and MALDI-MS detection.

\section{RESULTS AND DISCUSSION}

Combining MALDI-MS Signal Amplification by Mass Barcoded AuNPs with Immunomagnetic Separation: Optimization and Validation. To obtain the best signal amplification, several parameters of the proposed AuNPs/ immunomagnetic separation based approach have been optimized, including the choice of immunomagnetic support and protein linker, as described in SI-5 and SI-6. Analysis of antibovine $\beta$-lac B IgG Abs was chosen as a model system for the protocol optimization. Antibovine $\beta$-lac B Abs could bind with both protein A/G@MBs and $\beta$-lac B-coated AuNPs $(\beta$-lac $\mathrm{B} @$ AuNPs) probe forming a sandwich structure and then be detected through MALDI-MS analysis of corresponding mass barcode, as shown in Figure S4 of SI-4. The validity and sensitivity of the method were also evaluated based on this model. 
First of all, the amount ratio between mass barcode and protein linker for AuNPs surface modification needed the optimization. Excessive mass barcode amount in comparison to the protein linker could negatively affect the protein conjugation efficiency. In contrast, insufficient amount of mass barcode would lead to limited signal amplification effect. To find an optimal mass barcode/protein linker ratio, several identical solutions containing $1 \mathrm{nM}$ of antibovine $\beta$-lac B IgG Abs in $300 \mu \mathrm{L}$ of $10 \mathrm{mM} \mathrm{PB}$ were analyzed separately with the fixed amount of protein A/G@MBs $(100 \mu \mathrm{g})$ and $20 \mu \mathrm{L}$ of $\beta$ lac $\mathrm{B} @ A u N P s$ prepared with various mass barcode-to-protein linker amount ratios $(\mathrm{n} / \mathrm{m})$ ranging from $95: 5$ to $75: 25$. The amount of MBs was much more excessive in comparison to the amount of IgG Abs. According to the protocol, ${ }^{16}$ provided by the manufacturer of protein A/G@MBs, the chosen amount was enough to fix $4 \mu \mathrm{g}$ of IgG Abs. Therefore, most of the antibovine $\beta$-lac B IgG Abs should be immobilized on the MBs. The amount of $\beta$-lac B@AuNPs was chosen to match the amount of IgG Abs. For semiquantitative comparison, internal standard-coated AuNPs (IS@AuNPs) and 2,5-dihydroxybenzoic acid (DHB) were deposited together with the concentrated sample on a MALDI target plate for MS detection, as discussed in SI-4 and SI-7. Mass barcode 2 (Mbc2) was used as the IS, which has similar molecular structure as the signal reporter, $\mathrm{Mbcl}$, coated on AuNPs to ensure similar ionization efficiency during MALDI-MS analysis. The relative intensity of the mass barcode to IS was considered for the evaluation of the signal amplification effect during the detection of antibovine $\beta$ lac B Abs. From the recorded MALDI mass spectra, Mbc1 relative intensity reached the highest value, when the mass barcode-to-protein linker ratio was 85:15 (Figure S6(a) and (b) in SI-8). Therefore, the oligomer mixture composed of $85 \%$ mass barcode and $15 \%$ protein linker was selected to functionalize AuNPs for all the following immunoassays.

With the proposed strategy, the semiquantification of antibovine $\beta$-lac B IgG Abs was further performed, as described in SI-4. The method sensitivity was accessed by determining the LOD of antibovine $\beta$-lac B IgG Abs. Following the optimized conditions, both the amounts of the $\beta$-lac B@AuNPs probes and protein $\mathrm{A} / \mathrm{G} @ \mathrm{MBs}$ were kept constant at $300 \mathrm{fmol}$ and $100 \mu \mathrm{g}$ respectively, while the concentration of target Abs were decreased from $1 \mathrm{pM}$ to $30 \mathrm{fM}$. Figure 2 shows the obtained mass spectra of $\mathrm{Mbcl}$ and IS with intensities normalized to the intensity of IS. As expected, the relative intensity of $\mathrm{Mbcl}$ gradually decreased, as the concentration of Abs decreased. Due to the signal amplification effect, the lowest concentration of antibovine $\beta$-lac B IgG Abs that can be analyzed with a good reproducibility was around $100 \mathrm{fM}(18$ $\mathrm{pg} / \mathrm{mL}$ ). Once the concentration was lowered to $70 \mathrm{fM}$, the signal of $\mathrm{Mbcl}$ was hardly detected with $\mathrm{S} / \mathrm{N}$ around or less than 3 (data not shown).

In the negative control experiment using $\kappa$-casein@AuNPs and antibovine $\beta$-lac B Abs, only IS signal was observed in Figure 2e. Thanks to the oligomer coating of AuNPs and washing step applied, the obtained spectrum demonstrated clearly the high specificity of the system. Successful suppression of nonspecific interactions by the ethylene glycol oligomers ${ }^{17}$ was also confirmed by the optical estimation of the color difference and UV-vis absorption measurement of the supernatant eluted from the MBs by acidic solution when analyzing antibovine $\beta$-lac $\mathrm{B}$ Abs at high concentration ( $1 \mathrm{nM})$ or blank sample with $\beta$-lac B@AuNPs and $\kappa$-casein@AuNPs, as shown in Figure S7(a) and (c) in SI-9.

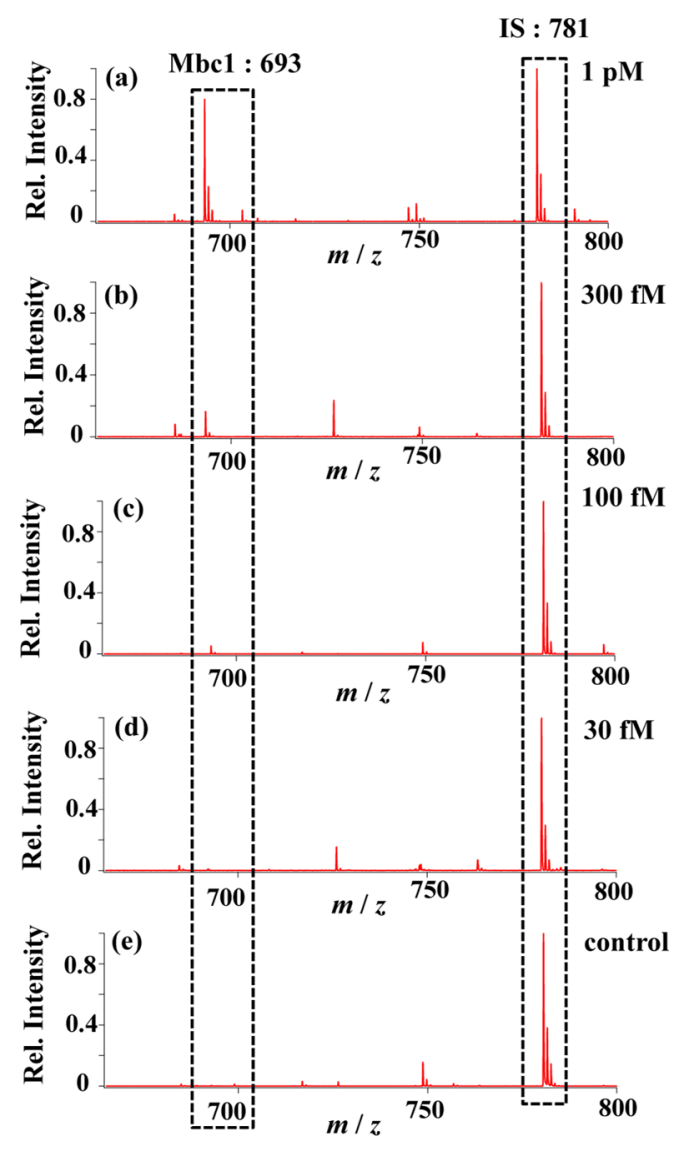

Figure 2. Detection of antibovine $\beta$-lac B IgG Abs by mass barcoded AuNPs-based MALDI-MS signal amplification method combined with immunomagnetic separation. MALDI mass spectra obtained for the analyses of (a) $1 \mathrm{pM}$, (b) $300 \mathrm{fM}$, (c) $100 \mathrm{fM}$, and (d) $30 \mathrm{fM}$ of antibovine $\beta$-lac B Abs. (e) $1 \mathrm{pM}$ of antibovine $\beta$-lac B Abs analyzed with $\kappa$-casein conjugated AuNPs as a negative control. Conditions: Abs in $300 \mu \mathrm{L}$ of $10 \mathrm{mM} \mathrm{PB}, 100 \mu \mathrm{g}$ of protein A/G@MBs, $300 \mathrm{fmol}$ AuNPs, 85:15 of $\mathrm{Mbcl}$ : protein linker ratio; Rel.: relative.

Multiplex Allergy CRD. The mass barcode-based MALDIMS signal amplification strategy was further applied to cow's milk allergy CRD. In this case, the immunoaffinity reactions between specific IgE Abs from the patient's blood serum and allergenic proteins were detected via the observation of amplified mass barcodes signals. Antihuman IgE Abs were immobilized onto protein A/G@MBs to selectively extract human IgE Abs from the blood serum of the patient. A large amount of magnetic immunosupport carrying antihuman IgE Abs could be prepared at once with the commercial protocol ${ }^{16}$ and divided into equal portions for all the following diagnostic steps, $100 \mu \mathrm{g}$ per each analysis. This amount of antihuman IgEprotein A/G@MBs was chosen to be largely in excess in comparison to the total IgE Abs in patient's blood serum. The total IgE Abs concentration in the original blood serum of the milk allergic patient was detected as $2150 \pm 160 \mathrm{ng} / \mathrm{mL}$ (10 $\mathrm{nM}$, or $900 \pm 70 \mathrm{IU} / \mathrm{mL}, n=3$ ) by classical commercial ELISA. $^{15}$ Initial volume of the blood serum was diluted continuously with $10 \mathrm{mM} \mathrm{PB}$ and, therefore varied depending on the dilution factor: for example, $50 \mu \mathrm{L}$ were for 10 times dilution, $10 \mu \mathrm{L}$ for 50 times dilution, and $1 \mu \mathrm{L}$ for the dilution 500 times and higher. Then, $300 \mu \mathrm{L}$ of the samples diluted were analyzed separately to evaluate the diagnosis sensitivity as described below. 
First, diagnosis was performed with individual AuNPs probes, Mbc1-coded lactoferrin@AuNPs, Mbc2-coded BSA@ AuNPs or Mbc3-coded $\beta$-lac B@AuNPs using the protocol, illustrated in Figure 1. Among the three whey proteins, BSA@ AuNPs and lactoferrin@AuNPs displayed the binding with the patient's IgE Abs on MBs based on the corresponding observation of $\mathrm{Mbc1}$ and $\mathrm{Mbc2}$ peaks in MS spectra (Figure 3). The $\mathrm{Mbcl}$ and $\mathrm{Mbc} 2$ signals were still detectable even when
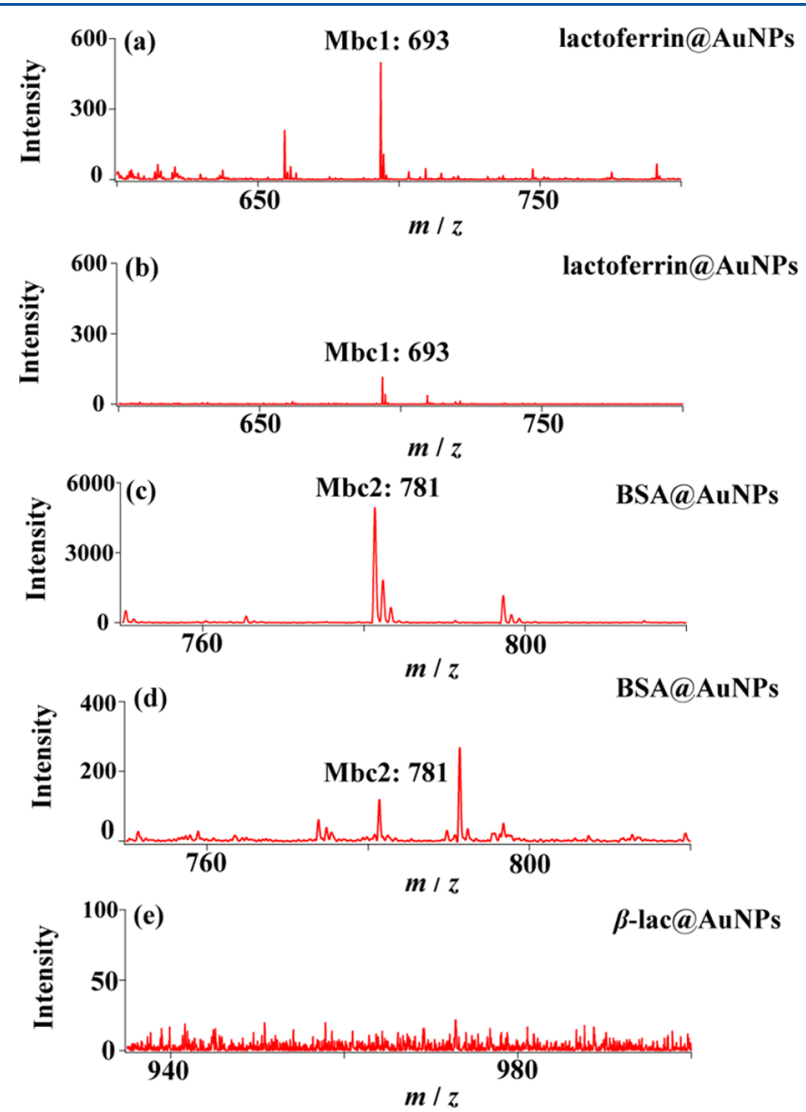

Figure 3. Diagnosis of cow's milk allergy with individual AuNPs probes combined with immunomagnetic separation. MALDI mass spectra of $\mathrm{Mbcl}$ for lactoferrin allergy diagnosis with the patient's serum diluted (a) 30000 and (b) 100000 times. MALDI mass spectra of Mbc2 for BSA allergy diagnosis with the patient's blood serum diluted (c) 10000 and (d) 30000 times. (e) Mass spectrum obtained using the Mbc3 coded $\beta$-lac@AuNPs and 10 times diluted patient's serum. Conditions: diluted sample volume of $300 \mu \mathrm{L}, 100 \mu \mathrm{g}$ of antihuman IgE-protein A/G@MBs and 300 fmol AuNPs probes.

the blood serum was diluted 100000 and 30000 times, as shown in Figure $3 \mathrm{~b}$ and $\mathrm{d}$, respectively. Considering that the original total concentration of the $\mathrm{IgE} \mathrm{Abs}$ in the blood serum was around $10 \mathrm{nM}(900 \mathrm{IU} / \mathrm{mL})$, lactoferrin- and BSA-specific $\operatorname{IgE}$ Abs were still detectable with the total IgE Abs concentrations of 100 and $300 \mathrm{fM}$ (18 and $54 \mathrm{pg} / \mathrm{mL}$ or 7.5 and $22.5 \mathrm{mIU} /$ $\mathrm{mL}$ ), respectively. Owing to such sensitivity, less than $1 \mu \mathrm{L}$ of original patient's blood serum could be enough for the allergy CRD based on MS signal amplification.

In contrast, no signal of $\mathrm{Mbc} 3$ at $\mathrm{m} / z 957$ was observed for $\beta$-lac B@AuNPs even with the 10 times diluted patient's blood serum, (Figure 3e), indicating that the patient should not be allergic to bovine $\beta$-lac, while he may have an allergy to BSA and lactoferrin.
To check the feasibility of the present strategy for multiplex analysis, the CRD of cow's milk allergy was performed with various AuNPs probes simultaneously in a single run. For this purpose, same amounts of the three kinds of AuNPs probes ( $300 \mathrm{fmol}$ each) were mixed together to react with the patient's IgE Abs extracted on the MBs. As each kind of AuNPs probe binds with its specific IgE Abs from the patient's serum without competition, the experimental parameters, for example, amount of individual AuNPs probes, were kept the same as those in individual allergen identification. As expected, for patient's blood serum diluted 30000 times, both signals of Mbc1 for lactoferrin specific IgE Abs and Mbc2 for BSA specific IgE Abs were detected, but without $\mathrm{Mbc} 3$ signal for $\beta$-lac B specific IgE Abs, as shown in Figure 4(a1). When the serum was diluted by
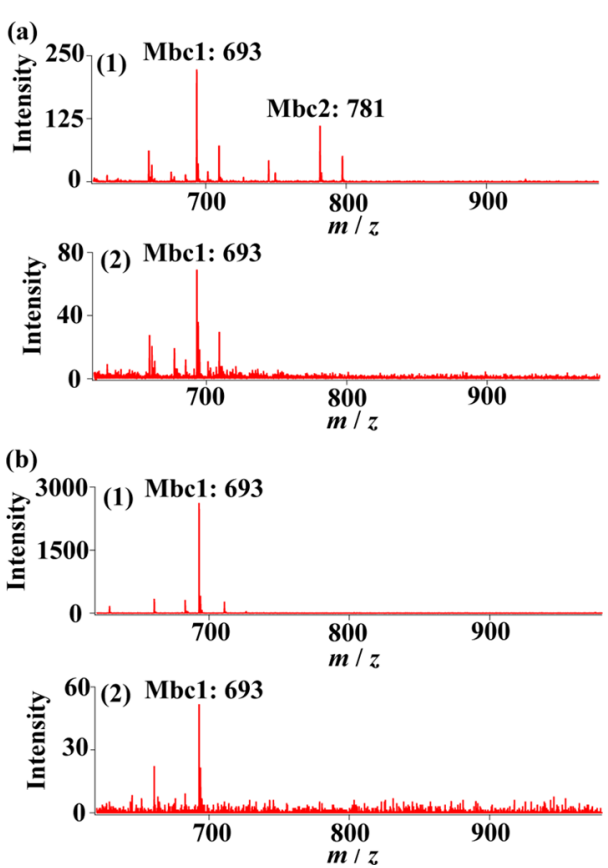

Figure 4. (a) Multiplex diagnosis of lactoferrin allergy ( $\mathrm{Mbc1}$ ), BSA allergy ( $\mathrm{Mbc} 2$ ), and $\beta$-lac $\mathrm{B}$ allergy $(\mathrm{Mb} 3)$ by AuNPs probes combined with immunomagnetic separation. MALDI mass spectra obtained with the patient's blood serum diluted (1) 30000 and (2) 100000 times. (b) Multiplex diagnosis of $\alpha$-casein (Mbc1 coded), $\beta$-casein (Mbc2 coded), and $\kappa$-casein (Mbc3 coded) allergies. [(1) and (2)] MALDI mass spectra obtained with the patient's blood serum diluted (1) 50 and (2) 1000 times. Conditions: diluted sample volume of $300 \mu \mathrm{L}, 100$ $\mu \mathrm{g}$ of antihuman IgE coated MBs, and $300 \mathrm{fmol}$ of each kind of AuNPs probes.

100000 times, the Mbc2 signal could not be observed any more, while $\mathrm{Mbcl}$ was still detectable (Figure 4(a2)), in accordance with the results obtained using individual AuNPs probes. These results demonstrate that the multiplex diagnosis would not sacrifice detection sensitivity, but could provide a gain in time and reagent consumption.

The same protocol was further applied for the multiplex diagnosis of allergy to caseins. $\mathrm{Mbcl}$ mass barcode was used for $\alpha$-casein@AuNPs, while Mbc2 and Mbc3 mass barcodes were utilized for $\beta$ - and $\kappa$-casein@AuNPs probes. With 50 times and 1000 times diluted patient's serum, only $\mathrm{Mbcl}$ was observed, demonstrating that the patient is solely allergic to $\alpha$-casein. In comparison with lactoferrin and BSA, the Mbc1 signal from $\alpha$ casein conjugated AuNPs probes was observed at LOD level with 1000 times diluted serum, as shown in Figure 4(b2). 
These data reveal that the amount of $\alpha$-casein specific IgE Abs existing in the blood serum is approximately 30 and 100 times lower than the amount of BSA and lactoferrin specific IgE Abs, respectively.

These results are in good accordance with those, previously obtained by IACE-UV/MS strategy and classical ELISA protocol, ${ }^{15}$ confirming the reliability of the present procedure. This diagnostic approach is qualitative, but provides the possibility to make a conclusion about the relative abundance of individual allergen specific IgE Abs coexisting in the patient's blood serum: specific IgE Abs to lactoferrin > IgE Abs to BSA > $\operatorname{IgE} \mathrm{Abs}$ to $\alpha$-casein. Such a comparison is feasible, since the diagnosis is only based on the observation of mass barcode molecules, which have similar molecular structures and comparable sensitivities during MALDI-MS analysis, as shown in Figure S8 in SI-10.

Comparison with Existing Approaches for Allergy Diagnosis. Different from the previous work based on IACE$\mathrm{UV} /-\mathrm{MS}$, this strategy aims at sensitively detecting specific $\operatorname{IgE}$ Abs in the allergic patient's blood serum with a set of known allergenic proteins. With the three mass-barcoded and allergenconjugated AuNPs, we have demonstrated the proof-of-concept of this strategy for qualitative multiplex allergy CRD. A high throughput multiplex diagnosis can be potentially realized by preparing more AuNPs modified with different mass barcode molecules and allergenic proteins. The high resolution of MS detection affords a wide choice of PEG based mass barcodes to perform the analysis of specific $\operatorname{IgE} \mathrm{Abs}$ against various allergens within a single assay.

The arbitrarily selected mass barcodes, easily controllable MBs in tube and sensitive MS detection allow performing multiplexed allergy diagnosis in a simple manner, avoiding the use of spatially resolved architectures, such as microarray systems, ${ }^{11,12}$ costly fluorescent ${ }^{18}$ or enzyme labels, ${ }^{19}$ and capillary electrophoresis that needs laborious preloading of immunoaffinity MBs and coupling with MS detection of the collected CE fractions for simultaneous antigen identification instead of UV analysis. ${ }^{15}$ Owing to the signal amplification procedure, the allergy diagnosis sensitivity has been enhanced to a level, which is 13 times better than that obtained with IACE-UV analysis ${ }^{15}$ and comparable to previously reported results employing nanocrystal clusters ${ }^{20}$ or nanoparticle hybrid probes. $^{21}$ In another case, surface plasmon resonance signals from AuNPs have been shown to provide ultrasensitive total IgE quantification with LOD of only $0.18 \mathrm{pg} / \mathrm{mL}(0.075 \mathrm{mIU} /$ $\mathrm{mL}){ }^{22}$ However, the method only provided quantification of the standard IgE solution instead of allergy CRD using patient's blood serum. Due to the high sensitivity, our method requires extremely low amount (3-30 $\mathrm{nL}$ in theory) of patient's blood serum in contrast to $20-100 \mu \mathrm{L}$ of blood serum consumption by commercial ELISA or other developed methods and $2 \mu \mathrm{L}$ by microarray chip with DLC solid support ${ }^{23}$ or IACE-UV/MS approach. $^{15}$

In addition, another three specific features distinguish this analysis platform from previously developed Au particle-based MS signal amplification for immunoassay performed on gold chips. ${ }^{3,4}$ First, MBs as the immunosupport with large surface area are commercially available with a wide variety of surface functionalization. They provide great flexibility for immunoassay design in effective and simple manner without complex and time-consuming surface conjugation processes for the gold chip. Second, the immune reactions may be faster in bulk solution with stirring in comparison with one on a fixed gold surface, facilitating sample handling and accelerating the immunobinding process. Third, deposition of concentrated AuNPs together with IS on the MALDI plate can provide more homogeneous MS analysis and allow avoiding spot-to-spot variation during the direct detection on the gold chip or complicated concentration process of AuNPs by centrifugation after their detachment from the gold chip.

All in all, due to the simplicity, tiny sample consumption and high sensitivity, the present technique can be a helpful tool for ultratrace analysis during clinical allergy diagnosis.

\section{CONCLUSIONS}

In this study, we have constructed a MALDI-MS signal amplification system based on mass-barcoded AuNPs and immunomagnetic separation. We have applied this system to enhance the sensitivity of allergy CRD. For diagnostic purpose, the patient's IgE Abs were first extracted from the blood serum by antihuman IgE Abs modified MBs, and then probed by different mass-barcoded and allergenic proteins-conjugated AuNPs. The immunobinding events between the patient's IgE Abs and allergens were reported by the corresponding mass barcodes. High sensitivity and potential for multiplexing allergy CRD were successfully demonstrated with down to $1 \mu \mathrm{L}$ of the initial patient's blood serum, that could be diluted up to 100000 times prior to the analysis. Besides the food allergen diagnosis, we believe that this system can be easily modified and applied to many other immunoassays with a vast range of target analytes.

\section{ASSOCIATED CONTENT}

\section{S Supporting Information}

The Supporting Information is available free of charge on the ACS Publications website at DOI: 10.1021/acs.analchem.6b01142.

Information about used materials and reagents; details about the synthesis of AuNPs and proteins@AuNPs; semiquantification of antibovine $\beta$-lac B Abs; optimization of the magnetic support, protein linker and the amount ratio between mass barcode and the chosen protein linker for AuNPs surface functionalization; verification of $\mathrm{Mbcl}$ and protein linker on AuNPs by MALDI MS; mass spectra of pure $\beta$-lac B and $\beta$-lac B conjugated on Mbcl-coded AuNPs ( $\beta$-lac B@AuNPs); mass spectra of Mbc1-coded AuNPs with and without DHB; UV-vis absorption measurements of the supernatant; MALDI-MS detection sensitivity of the three mass barcodes (PDF).

\section{AUTHOR INFORMATION}

\section{Corresponding Author}

*E-mail: hubert.girault@epfl.ch.

\section{Notes}

The authors declare no competing financial interest.

\section{ACKNOWLEDGMENTS}

X.Z. would like to acknowledge the China Scholarship Council for her Ph.D. study scholarship.

\section{REFERENCES}

(1) Qiu, F.; Jiang, D. W.; Ding, Y. B.; Zhu, J.; Huang, L. L. Angew. Chem., Int. Ed. 2008, 47, 5009-5012. 
(2) Qiu, F.; Gu, K.; Yang, B.; Ding, Y. B.; Jiang, D. W.; Wu, Y.; Huang, L. Q. L. Talanta 2011, 85, 1698-1702.

(3) Lee, J. R.; Lee, A.; Kim, S. K.; Kim, K. P.; Park, H. S.; Yeo, W. S. Angew. Chem., Int. Ed. 2008, 47, 9518-9521.

(4) Hong, S. H.; Kim, J. I.; Kang, H.; Yoon, S.; Kim, D. E.; Jung, W.; Yeo, W. S. Chem. Commun. (Cambridge, U. K.) 2014, 50, 4831-4834. (5) Yan, B.; Kim, S. T.; Kim, C. S.; Saha, K.; Moyano, D. F.; Xing, Y. Q.; Jiang, Y.; Roberts, A. L.; Alfonso, F. S.; Rotello, V. M.; Vachet, R. W. J. Am. Chem. Soc. 2013, 135, 12564-12567.

(6) Yang, J. H.; Chaurand, P.; Norris, J. L.; Porter, N. A.; Caprioli, R. M. Anal. Chem. 2012, 84, 3689-3695.

(7) Nam, J.; Yoo, M.; Yeo, W. S. BioChip J. 2015, 9, 124-130.

(8) Hochwallner, H.; Schulmeister, U.; Swoboda, I.; Spitzauer, S.; Valenta, R. Methods 2014, 66, 22-33.

(9) Fiocchi, A.; Schunemann, H. J.; Brozek, J.; Restani, P.; Beyer, K.; Troncone, R.; Martelli, A.; Terracciano, L.; Bahna, S. L.; Rance, F.; Ebisawa, M.; Heine, R. G.; Assa'ad, A.; Sampson, H.; Verduci, E.; Bouygue, G. R.; Baena-Cagnani, C.; Canonica, W.; Lockey, R. F. J. Allergy Clin. Immunol. 2010, 126, 1119-U1197.

(10) http://www.abnova.com.

(11) http://www.phadia.com/en/Products/Allergy-testing-products/ ImmunoCAP-ISAC/.

(12) Monroe, M. R.; Reddington, A. P.; Collins, A. D.; LaBoda, C.; Cretich, M.; Chiari, M.; Little, F. F.; Unlu, M. S. Anal. Chem. 2011, 83, 9485-9491.

(13) Hahn, Y. K.; Jin, Z.; Kang, J. H.; Oh, E.; Han, M. K.; Kim, H. S.; Jang, J. T.; Lee, J. H.; Cheon, J.; Kim, S. H.; Park, H. S.; Park, J. K. Anal. Chem. 2007, 79, 2214-2220.

(14) Monroe, M. R.; Daaboul, G. G.; Tuysuzoglu, A.; Lopez, C. A.; Little, F. F.; Unlu, M. S. Anal. Chem. 2013, 85, 3698-3706.

(15) Gasilova, N.; Girault, H. H. Anal. Chem. 2014, 86, 6337-6345.

(16) https://tools.thermofisher.com/content/sfs/manuals/ MAN0011761_Pierce_Protein_AG_Mag_Bead_UG.pdf.

(17) Prime, K. L.; Whitesides, G. M. Science 1991, 252, 1164-1167.

(18) Cerecedo, I.; Zamora, J.; Shreffler, W. G.; Lin, J.; Bardina, L.; Dieguez, M. C.; Wang, J.; Muriel, A.; de la Hoz, B.; Sampson, H. A. J. Allergy Clin. Immunol. 2008, 122, 589-594.

(19) Fall, B. I.; Eberlein-Konig, B.; Behrendt, H.; Niessner, R.; Ring, J.; Weller, M. G. Anal. Chem. 2003, 75, 556-562.

(20) Yao, J. J.; Han, X. G.; Zeng, S.; Zhong, W. W. Anal. Chem. 2012, $84,1645-1652$.

(21) Li, H.; Qiang, W. B.; Vuki, M.; Xu, D. K.; Chen, H. Y. Anal. Chem. 2011, 83, 8945-8952.

(22) Kim, S.; Lee, J.; Lee, S. J.; Lee, H. J. Talanta 2010, 81, 17551759.

(23) Suzuki, K.; Hiyoshi, M.; Tada, H.; Bando, M.; Ichioka, T.; Kamemura, N.; Kido, H. Anal. Chim. Acta 2011, 706, 321-327. 